\title{
SETS OF DISJOINT ELEMENTS IN FREE PRODUCTS OF LATTICE-ORDERED GROUPS
}

\author{
WAYNE B. POWELL AND CONSTANTINE TSINAKIS
}

(Communicated by Bhama Srinivasan)

\begin{abstract}
We show that for every infinite cardinal number $m$ there exist two totally ordered abelian groups whose free product in any nontrivial variety of lattice-ordered groups has a disjoint set of cardinality $m$. This answers problem 10.7 of [13] and extends the results in [12]. We further prove that for the variety of abelian lattice-ordered groups or the variety of all lattice-ordered groups, the free product of two nontrivial members of the variety will always contain an infinite disjoint set.
\end{abstract}

1. Introduction. Let $m$ be an infinite cardinal. An $l$-group (lattice-ordered group) $G$ is said to satisfy the $m$-disjointness condition if for every $a \in G$ and every $S \subseteq G$ it follows that $|S|<m$ whenever $a \notin S$ and $x \wedge y=a$ for all distinct $x, y \in G$. Such a set $S$ is said to be disjoint (with respect to $a$ ). Note that every group translation of an $l$-group is a lattice automorphism and hence one only need consider disjoint sets with respect to the group identity in deciding the failure or satisfaction of the $m$-disjointness condition.

The question of the preservation of the $m$-disjointness condition for free products in varieties of $l$-groups was addressed in [12]. It was shown there that if $\mathscr{A}$ is the variety of abelian $l$-groups, then the $\mathscr{A}$-free product of two totally ordered members of $\mathscr{A}$ can have arbitrarily large cardinality. It was further shown that if $\mathscr{U}$ is any nontrivial variety of $l$-groups, then $\mathscr{U}$-free products do not preserve the $m$-disjointness condition whenever $m$ is regular but not weakly compact, countable, or singular. The proof of the latter result assumes the existence of an $m$-Souslin tree whenever $m$ is a regular cardinal that is not weakly compact and is based on a related lattice theoretic result of Adams and Kelly [1]. Thus the following problem presents itself.

PROBLEM 1 . Let $\mathscr{U}$ be any nontrivial variety of $l$-groups. Do $\mathscr{U}$-free products fail to preserve the $m$-disjointness condition for all infinite cardinals $m$ and, if so, can this failure be decided on the basis of the standard axioms of set theory?

The first objective of this paper is to provide an affirmative answer to this problem. More specifically, Theorem 4 asserts that given an infinite cardinal $m$, there exist totally ordered abelian groups $G_{1}$ and $G_{2}$ of cardinality $m$ such that for any nontrivial variety $\mathscr{U}$ of $l$-groups the $\mathscr{U}$-free product of $G_{1}$ and $G_{2}$ has a disjoint set of cardinality $m$. This result is actually an easy consequence of a more general

Received by the editors July 1, 1987 and, in revised form, February 1988.

1980 Mathematics Subject Classification (1985 Revision). Primary 06F15, 06F20; Secondary $08 \mathrm{~B} 25$.

Research of the first author supported by NSF grant no. DMS-8401309.

Research of the second author supported by the Vanderbilt University Research Council. 
result relating disjoint sets in free products with certain chains in the free factors (Theorem 3).

Recall that the lattice of an $l$-group is a distributive lattice (see Bigard et al. [3, p. 19]). Furthermore, there is a close connection between free products in varieties of $l$-groups and free products in the class of distributive lattices (Franchello [4], Powell and Tsinakis [11]). Thus the theorem above is somewhat surprising when contrasted with the fact that free products in the variety of distributive lattices preserve the $m$-disjointness condition whenever the cardinal $m$ is singular or weakly compact (Adams and Kelly [1]).

A closely related problem is the following.

PROBLEM 2. Suppose that $\mathscr{U}$ is a nontrivial variety of $l$-groups. Does the $\mathscr{U}$-free product of $G_{1}$ and $G_{2}$ have an infinite disjoint set whenever $G_{1}$ and $G_{2}$ are nontrivial members of $\mathscr{U}$ ?

It has been recently shown in Glass [6] that Problem 2 has an affirmative answer in the special case $\mathscr{U}$ is the variety of all $l$-groups and $G_{1}$ and $G_{2}$ are countable. We show that Problem 2 has an affirmative answer for the variety of abelian $l$-groups and that of all $l$-groups (Theorem 7 ) without any restrictions on $G_{1}$ and $G_{2}$. A partial affirmative answer for an arbitrary $\mathscr{U}$ is provided by Theorem 8.

The aforementioned results would seem to indicate that, apart from trivial cases, free products never preserve disjointness conditions. However, this is not the case. For example, any $\mathscr{U}$-free $l$-group of rank $\geq 2$ satisfies the $\aleph_{1}$-disjointness condition and fails the $\aleph_{0}$-disjointness condition (see [12]). It follows that the $\mathscr{U}$-free product of two such $l$-groups preserved the $\aleph_{1}$-disjointness condition. It would be of interest to obtain general theorems along these lines.

Before we embark on the proof of the theorems, let us recall the concept of a free product and establish some notation.

Let $\mathscr{U}$ be a class of algebras of the same similarity type and let $\left(G_{i} \mid i \in I\right)$ be a family of members of $\mathscr{U}$. The $\mathscr{U}$-free product of the family is an algebra $G \in \mathscr{U}$, denoted by ${ }^{\mathscr{U}} \bigsqcup_{i \in I} G_{i}$, together with a family of injective homomorphisms $\left(a_{i}: G_{i} \rightarrow G \mid i \in I\right)$ such that

(i) $\bigcup_{i \in I} a_{i}\left(G_{i}\right)$ generates $G$;

(ii) if $H \in \mathscr{U}$ and $\left(\beta_{i}: G_{i} \rightarrow H \mid i \in I\right)$ is a family of homomorphisms, then there exists a (necessarily) unique homomorphism $\gamma: G \rightarrow H$ satisfying $\beta_{i}=\gamma \alpha_{i}$ for all $i \in I$.

We will always identify each free factor $G_{i}$ with its image $\alpha_{i}\left(G_{i}\right)$ in ${ }^{\mathscr{U}} \bigsqcup_{i \in I}$ and thus view each $\alpha_{i}$ as the inclusion map.

Given a family $\left(G_{i} \mid i \in I\right)$ in a variety $\mathscr{U}$, it is well known that ${ }^{\mathscr{U}} \bigsqcup_{i \in I} G_{i}$ exists if and only if there exists $H \in \mathscr{U}$ so that each $G_{i}$ can be embedded in $H$. This condition is clearly satisfied when $\mathscr{U}$ is a variety of $l$-groups, for then one can let $H$ be the (cardinal) product of the $l$-groups $G_{i}$. For information on properties of l-group free products it is best to first look at [13].

In what follows we use $\mathscr{U}$ to denote an arbitrary nontrivial variety of $l$-groups. Two varieties which have special significance and are given distinguished notation are the variety of abelian $l$-groups, denoted by $\mathscr{A}$, and the variety of all $l$-groups, denoted by $\mathscr{L}$. It is well-known that $\mathscr{A} \subseteq \mathscr{U} \subseteq \mathscr{L}$ (Weinberg [14]).

Group operations will be written multiplicatively throughout. The cardinal sum $\boxplus_{i \in I} H_{i}$ of a family $\left(H_{i} \mid i \in I\right)$ of $l$-groups is the group direct sum, $\bigoplus_{i \in I} H_{i}$, endowed 
with the componentwise lattice order. If $x$ is an element of an $l$-group, then its positive part, $x \vee 1$, is denoted by $x^{+}$. The reader may consult Bigard et al. [3] and Glass [5] for elementary concepts not defined here.

2. Construction of the disjoint sets. If $\Omega$ is a chain, we denote by $A(\Omega)$ the $l$-group (under the pointwise order) of all order preserving permutations of $\Omega$. An $l$-subgroup $G$ of $A(\Omega)$ is referred to as an $l$-permutation group. When $G$ is identified as a set of order preserving permutations from $A(\Omega)$, it is labelled $(G, \Omega)$. $\Omega$ is said to be doubly homogeneous if $A(\Omega)$ is doubly transitive: whenever $\alpha_{1}<\alpha_{2}$ and $\beta_{1}<\beta_{2}$ are elements in $\Omega$, there is a $g \in A(\Omega)$ such that $g\left(a_{1}\right)=\beta_{1}$ and $g\left(a_{2}\right)=\beta_{2}$. It is not hard to see that a doubly homogeneous chain is dense, that is, given $a_{1}<a_{2}$ in $\Omega$, there is $a_{3} \in \Omega$ with $a_{1}<a_{3}<a_{2}$.

The following fundamental result due to Holland [7] (see Glass [5, p. 41 and p. 240]) will be used in some of the subsequent proofs.

LEMMA 1. Every l-group can be embedded in $A(\Omega)$ for a suitable chain $\Omega$. In fact, $\Omega$ can be chosen to be doubly homogeneous.

LEMMA 2. Let $a_{1}, a_{2}, b_{1}, b_{2}$ be positive elements of an l-group $G$ such that $a_{2}^{2}<$ $a_{1}$ and $b_{1}^{2}<b_{2}$. If

$$
x_{i}=\left[\left(a_{i}^{2} b_{i}^{-1}\right) \wedge\left(b_{i}^{2} a_{i}^{-1}\right)\right]^{+}
$$

for $i=1,2$, then $x_{1} \wedge x_{2}=1$.

PROOF. In view of Lemma 1 , we may view $G$ as an $l$-permutation group $(G, \Omega)$ for a suitable chain $\Omega$. Since $a_{1}^{2} b_{1}^{-1} \geq a_{2}^{2} b_{2}^{-1}$ and $b_{2}^{2} a_{2}^{-1} \geq b_{1}^{2} a_{1}^{-1}$, it follows that

$$
1 \leq x_{1} \wedge x_{2} \leq\left(b_{1}^{2} a_{1}^{-1}\right)^{+} \wedge\left(a_{2}^{2} b_{2}^{-1}\right)^{+},
$$

and hence it is sufficient to prove that the right-hand side of the inequality is equal to 1 . This is of course equivalent to showing that for all $\omega \in \Omega$,

$$
\min \left\{b_{1}^{2} a_{1}^{-1}(\omega), a_{2}^{2} b_{2}^{-1}(\omega)\right\} \leq \omega .
$$

To this end, let $\omega \in \Omega$ and suppose that $b_{1}^{2} a_{1}^{-1}(\omega)>\omega$, i.e., $a_{1}^{-1}(\omega)>b_{1}^{-2}(\omega)$. Now by hypothesis, $a_{2}^{-2}(\omega) \geq a_{1}^{-1}(\omega)$ and $b_{1}^{-2}(\omega) \geq b_{2}^{-1}(\omega)$. It follows that $a_{2}^{-2}(\omega)>$ $b_{2}^{-1}(\omega)$ or $a_{2}^{2} b_{2}^{-1}(\omega)<\omega$.

The next result shows that disjoint sets in a free product can be built from suitable chains in the free factors. Let $G$ be an $l$-group and let $m$ be a cardinal number. A chain $\left(a_{\lambda} \mid \lambda<m\right)$ in $G$ of length $m$ is said to be an admissible ascending (descending) chain if for all $\lambda<\mu<m, 1<a_{\lambda}^{2}<a_{\mu}\left(1<a_{\mu}^{2}<a_{\lambda}\right)$.

THEOREM 3. Let $m$ be an infinite cardinal. Suppose that $G_{1}$ and $G_{2}$ are abelian l-groups such that $G_{1}$ has an admissible descending chain of length $m$ and $G_{2}$ has an admissible ascending chain of length $m$. Then for every nontrivial variety $\mathscr{U}$ of l-groups, $G_{1}{ }^{\mathscr{U}} \bigsqcup G_{2}$ has a disjoint set of cardinality $m$.

ProOF. Let $\left(a_{\lambda} \mid \lambda<m\right)$ be an admissible descending chain in $G_{1}$, and $\left(b_{\lambda} \mid \lambda<\right.$ $m$ ) an admissible ascending chain in $G_{2}$. Consider the elements

$$
x_{\lambda}=\left[\left(a_{\lambda}^{2} b_{\lambda}^{-1}\right) \wedge\left(b_{\lambda}^{2} a_{\lambda}^{-1}\right)\right]^{+} \quad(\lambda<m)
$$

in $G_{1} \mathscr{L} \sqcup G_{2}$ and the set $S_{\mathscr{L}}=\left\{x_{\lambda} \mid \lambda<m\right\}$. 
We have remarked that $\mathscr{A} \subseteq \mathscr{U} \subseteq \mathscr{L}$ and hence the identity mappings on $G_{1}$ and $G_{2}$ induce the epimorphisms

$$
\phi_{\mathscr{U}}: G_{1}^{\mathscr{L}} \sqcup G_{2} \rightarrow G_{1}^{\mathscr{U}} \sqcup G_{2}
$$

and

$$
\psi_{\mathscr{U}}: G_{1}^{\mathscr{U}} \sqcup G_{2} \rightarrow G_{1}^{\mathscr{A}} \sqcup G_{2} .
$$

The goal is to show that $S_{\mathscr{U}}=\left\{\phi_{\mathscr{U}}\left(x_{\lambda}\right) \mid \lambda<m\right\}$ is a disjoint set in $G_{1}{ }^{\mathscr{U}} \sqcup G_{2}$ of cardinality $m$. The proof below incorporates ideas from the proof of Theorem 2.1 of $[12]$.

To begin with, in view of Lemma 2, it is sufficient to prove that $S_{\mathscr{U}} \cap\{1\}=\varnothing$. Further, since every $S_{\mathscr{U}}$ is projected onto $S_{\mathscr{A}}$ by $\psi_{\mathscr{U}}$, we only need show that $S_{\mathscr{A}} \cap\{1\}=\varnothing$. To this end, we fix $\lambda<m$ and proceed to show that $\phi_{\mathscr{A}}\left(x_{\lambda}\right) \neq 1$ in $G_{1} \mathscr{A} \sqcup G_{2}$. Let $P_{i}$ be a prime subgroup of $G_{i}(i=1,2)$ such that $a_{\lambda} \notin P_{1}$ and $b_{\lambda} \notin P_{2}$. Consider the totally ordered groups $H_{i}=G_{i} / P_{i}(i=1,2)$ and let $\psi: G_{1}^{\mathscr{A}} \cup G_{2} \rightarrow H_{1}^{\mathscr{A}} \cup H_{2}$ be the epimorphism extending the natural projections. It will be sufficient to verify that $\psi \phi_{\mathscr{A}}\left(x_{\lambda}\right) \neq 1$ in $H_{1} \mathscr{A} \sqcup H_{2}$. Set $\psi\left(a_{\lambda}\right)=a$ and $\psi\left(b_{\lambda}\right)=b$. In view of the universal property of $\mathscr{A}$-free products, it will be enough to prove that the elements $a^{2} b^{-1}$ and $b^{2} a^{-1}$ in $H_{1} \oplus H_{2}$ are positive with respect to some total order on $H_{1} \oplus H_{2}$ extending the orders of $H_{1}$ and $H_{2}$. Now any partial order on a torsion-free abelian group can be extended to a total order (see for example Mura and Rhemtulla [9, §3.1]), and thus it will suffice to prove that there is a partial order on $H_{1} \oplus H_{2}$ whose cone contains the set $\left(H_{1} \boxplus H_{2}\right)^{+} \cup\left\{a^{2} b^{-1}, b^{2} a^{-1}\right\}$. It is easy to see that such a partial order exists if and only if for all $n_{1}, n_{2} \in \mathbf{Z}^{+} \backslash\{0\}$, the combination $\left(a^{2} b^{-1}\right)^{n_{1}}\left(a^{2} b^{-1}\right)^{n_{2}}$ is not negative in $H_{1} \boxplus H_{2}$. Thus suppose that $\left(a^{2} b^{-1}\right)^{n_{1}}\left(a^{-1} b^{2}\right)^{n_{2}} \leq 1$ in $H_{1} \boxplus H_{2}$ for some $n_{1}, n_{2} \in \mathbf{Z}^{+}$. Then $a^{2 n_{1}-n_{2}} \leq 1$ and $b^{2 n_{2}-n_{1}} \leq 1$ in $H_{1}$ and $H_{2}$, respectively. As $a>1$ in $H_{1}$ and $b>1$ in $H_{2}$, this implies $2 n_{1}-n_{2} \leq 0$ and $2 n_{2}-n_{1} \leq 0$ and so $n_{1}=n_{2}=0$.

We can now use Theorem 3 to give an affirmative answer to Problem 1.

THEOREM 4. Let $m$ be an infinite cardinal. There exists totally ordered abelian groups $G_{1}$ and $G_{2}$ of cardinality $m$ such that for any nontrivial variety $\mathscr{U}$ of $l$ groups, $G_{1} \mathscr{\text { Ü }} \cup G_{2}$ has a disjoint set of cardinality $m$.

PROOF. Let $\Lambda$ be a totally ordered set of cardinality $m$. Define

$$
G_{1}=\bigoplus_{\lambda \in \Lambda} G_{\lambda}^{(1)} \quad \text { and } \quad G_{2}=\bigoplus_{\lambda \in \Lambda} G_{\lambda}^{(2)}
$$

where $G_{1} \cap G_{2}=\{1\}$, and for each $\lambda \in \Lambda, G_{\lambda}^{(1)}$ and $G_{\lambda}^{(2)}$ are isomorphic to the totally ordered group $\mathbf{Z}$ of integers. Endow $G_{1}$ and $G_{2}$ with the lexicographic and the dual lexicographic order respectively. Thus if $x=x_{1} x_{2} \cdots x_{n}$ with $x_{i} \in G_{\lambda_{i}}^{(1)} \backslash\{1\}$ (respectively, $x_{i} \in G_{\lambda_{i}}^{(2)} \backslash\{1\}$ ) and $\lambda_{1}<\lambda_{2}<\cdots<\lambda_{n}$, then $x>1$ in $G_{1}$ iff $x_{1}>1$ in $G_{\lambda_{1}}^{(1)}\left(\right.$ and $x>1$ in $G_{2}$ iff $x_{n}>1$ in $G_{\lambda_{n}}^{(2)}$ ).

Now for each $\lambda \in \Lambda$, pick $a_{\lambda} \in\left(G_{\lambda}^{(1)}\right)^{+} \backslash\{1\}$ and $b_{\lambda} \in\left(G_{\lambda}^{(2)}\right)^{+} \backslash\{1\}$. It is readily seen that $\left(a_{\lambda} \mid \lambda \in \Lambda\right)$ is an admissible descending chain in $G_{1}$ and $\left(b_{\lambda} \mid \lambda \in \Lambda\right)$ is an admissible ascending chain in $G_{2}$. The result now follows from Theorem 3 since these two chains have length $m$. 
It would be of interest to determine whether Theorem 3 remains true when $G_{1}$ and $G_{2}$ are arbitrary $l$-groups in $\mathscr{U}$. The following result shows that this is the case if $\mathscr{U}=\mathscr{L}$.

THEOREM 5. Let $m$ be an infinite cardinal and let $\mathscr{U}=\mathscr{A}$ or $\mathscr{L}$. Suppose that $G_{1}$ and $G_{2}$ are l-groups in $\mathscr{U}$ such that $G_{1}$ has an admissible descending chain of length $m$ and $G_{2}$ has an admissible chain of length $m$. Then $G_{1}^{\mathscr{U}} \sqcup G_{2}$ has a disjoint set of cardinality $m$.

Proof. The case $\mathscr{U}=\mathscr{A}$ is found in Theorem 3. Thus suppose that $\mathscr{U}=\mathscr{L}$. Let $\left(a_{\lambda} \mid \lambda<m\right)$ be an admissible descending chain in $G_{1}$ and let $\left(b_{\lambda} \mid \lambda<m\right)$ be an admissible ascending chain in $G_{2}$. Define the subset $S_{\mathscr{L}}=\left\{x_{\lambda} \mid \lambda<m\right\}$ of $G=G_{1} \mathscr{L} \cup G_{2}$ as the proof of Theorem 3:

$$
x_{\lambda}=\left[\left(a_{\lambda}^{2} b_{\lambda}^{-1}\right) \wedge\left(b_{\lambda}^{2} a_{\lambda}^{-1}\right)\right]^{+} .
$$

In view of Lemma 2 , we only need show that $S_{\mathscr{L}} \cap\{1\}=\varnothing$. To this end, we fix $\lambda<m$ and proceed to show that $x_{\lambda} \neq 1$. By Lemma 1 , we may view $G$ as an $l$-permutation group $(G, \Omega)$, where $A(\Omega)$ is doubly transitive. Now $a_{\lambda}, b_{\lambda}>1$ and hence there exist $\omega_{1}, \omega_{2} \in \Omega$ such that $a_{\lambda}^{-1}\left(\omega_{1}\right)<\omega_{1}$ and $b_{\lambda}^{-1}\left(\omega_{2}\right)<\omega_{2}$. Thus, since $\Omega$ is doubly homogeneous, there exists $h \in A(\Omega)$ such that $h\left(\omega_{2}\right)=\omega_{1}$ and $h\left(b_{\lambda}^{-1}\left(\omega_{2}\right)\right)=a_{\lambda}^{-1}\left(\omega_{1}\right)$. We have $a_{\lambda}^{-1}\left(\omega_{1}\right)=c_{\lambda}^{-1}\left(\omega_{1}\right)<\omega_{1}$, where $c_{\lambda}=h b_{\lambda} h^{-1}$. It follows that

$$
a_{\lambda}^{2} c_{\lambda}^{-1}\left(\omega_{1}\right)=a_{\lambda}\left(\omega_{1}\right)>\omega_{1}
$$

and

$$
c_{\lambda}^{2} a_{\lambda}^{-1}\left(\omega_{1}\right)=c_{\lambda}\left(\omega_{1}\right)>\omega_{1}
$$

This shows that

$$
\left(a_{\lambda}^{2} c_{\lambda}^{-1}\right)^{+} \wedge\left(c_{\lambda}^{2} a_{\lambda}^{-1}\right)^{+}>1
$$

in $A(\Omega)$. Consider now the $l$-homomorphism $f: G \rightarrow A(\Omega)$ extending the inclusion map $f_{1}: G_{1} \rightarrow A(\Omega)$ and the $l$-homomorphism $f_{2}: G_{2} \rightarrow A(\Omega)$ defined by $f_{2}(g)=$ $h g h^{-1}$ for all $g \in G_{2}$. In view of $(*), f\left(x_{\lambda}\right)>1$ in $f(G)$ and hence $x_{\lambda}>1$ in $G$. This completes the proof of the theorem.

The remainder of the paper is concerned with Problem 2. Given an $l$-group $G$, we write $G^{\prime}$ for the $l$-ideal of $G$ generated by the derived subgroup.

THEOREM 6. Let $\mathscr{U}$ be a nontrivial l-group variety and let $G_{1}, G_{2}$ be l-groups in $\mathscr{U}$ such that $G_{1}^{\prime} \neq G_{1}$ and $G_{2}^{\prime} \neq G_{2}$. Then $G_{1} \mathscr{U}^{\mathscr{U}} \cup G_{2}$ has an infinite disjoint set.

ProOF. Let $x \in G_{1}^{+} \backslash G_{1}^{\prime}$ and $y \in G_{2}^{+} \backslash G_{2}^{\prime}$. For each $n \in \mathbf{Z}^{+} \backslash\{0\}$, let

$$
s_{n}=\left[\left(x^{-(n-1)} y\right) \wedge\left(y^{-1} x^{n}\right)\right]^{+}
$$

in $G=G_{1}^{\mathscr{U}} \sqcup G_{2}$. We claim that

$$
S=\left\{s_{n} \mid n \in \mathbf{Z}^{+} \backslash\{0\}\right\}
$$

is an infinite disjoint set in $G$.

To begin with, let $m, n \in \mathbf{Z}^{+} \backslash\{0\}$ with $m<n$. Then

$$
1 \leq s_{m} \wedge s_{n} \leq\left(y^{-1} x^{m}\right)^{+} \wedge\left(x^{-m} y\right)^{+}=1 \text {. }
$$


We complete the proof by showing that $s_{n} \neq 1$ for all $n$. Fix $n \in Z^{+} \backslash\{0\}$. Note that $G / G^{\prime}$ is a nontrivial abelian $l$-group whenever $G^{\prime} \neq G$. Furthermore, each abelian $l$-group is a subdirect product of totally ordered groups. Hence, for $i=1,2$, there exists an $l$-ideal $P_{i}$ of $G_{i}$ such that $G_{i} / P_{i}=H_{i}$ is a totally ordered abelian group, $x \notin P_{1}$, and $y \notin P_{2}$. As $\mathscr{A} \subseteq \mathscr{U}$, the natural projections $G_{1} \rightarrow H_{1}$ and $G_{2} \rightarrow$ $H_{2}$ induce an epimorphism $p: G_{1} \overline{\mathscr{\mathscr { C }}} \cup G_{2} \rightarrow H_{1} \mathscr{A} \cup H_{2}$. Thus it will suffice to show that $p\left(s_{n}\right) \neq 1$. This in turn is equivalent to showing that for all $n_{1}, n_{2} \in \mathbf{Z}^{+} \backslash\{0\}$, the element $\left(p(x)^{-(n-1)} p(y)\right)^{n_{1}}\left(p(y)^{-1} p(x)^{n}\right)^{n_{2}}$ is not negative in $H_{1} \boxplus H_{2}$. (See the proof of Theorem 3.) Thus suppose that $\left(p(x)^{-(n-1)} p(y)\right)^{n_{1}}\left(p(y)^{-1} p(x)^{n}\right)^{n_{2}} \leq 1$ in $H_{1} \boxplus H_{2}$ for some $n_{1}, n_{2} \in \mathbf{Z}^{+}$. Then

$$
p(x)^{n n_{2}-(n-1) n_{1}} \leq 1
$$

and

$$
p(y)^{n_{1}-n_{2}} \leq 1
$$

in $H_{1}$ and $H_{2}$ respectively. As $p(x)>1$ in $H_{1}$ and $p(y)>1$ in $H_{2}$, it follows that $n\left(n_{2}-n_{1}\right)+n_{1} \leq 0$ and $n_{2}-n_{2} \geq 0$. Thus $n_{1}=n_{2}=0$, as was to be shown.

We use Theorem 6 to provide an affirmative answer to Problem 2 when $\mathscr{U}=\mathscr{A}$ or $\mathscr{L}$.

THEOREM 7. If $\mathscr{U}=\mathscr{A}$ or $\mathscr{L}$, then the $\mathscr{U}$-free product of two nontrivial l-groups in $\mathscr{U}$ has an infinite disjoint set.

Proof. The case $\mathscr{U}=\mathscr{A}$ follows directly from Theorem 6 . Suppose that $\mathscr{U}=\mathscr{L}$ and let $G_{1}, G_{2}$ be two nontrivial $l$-groups in $\mathscr{L}$. Pick $x \in G_{1}^{+} \backslash\{1\}$ and $y \in G_{2}^{+} \backslash\{1\}$. For each $n \in \mathbf{Z}^{+} \backslash\{0\}$, let

$$
s_{n}=\left[\left(x^{-(n-1)} y\right) \wedge\left(y^{-1} x^{n}\right)\right]^{+}
$$

in $G=G_{1}{ }^{\mathscr{L}} \sqcup G_{2}$. We proceed to prove that

$$
S=\left\{s_{n} \mid n \in \mathbf{Z}^{+} \backslash\{0\}\right\}
$$

is an infinite disjoint set in $G$. It was observed in the proof of Theorem 6 that if $m \neq n$, then $s_{m} \wedge s_{n}=1$. Thus it will suffice to show that $s_{n} \neq 1$ for all $n$. Fix $n \in \mathbf{Z}^{+} \backslash\{0\}$ and view $G$ as an $l$-permutation group $(G, \Omega)$, where $\Omega$ is doubly homogeneous. Note that $x^{n}>x^{n-1}$ and $y>1$. Hence there are elements $\omega, \omega_{1} \in \Omega$ such that $x^{n}(\omega)>x^{n-1}(\omega)$ and $y\left(\omega_{1}\right)>\omega_{1}$. Choose $\omega_{2} \in A(\Omega)$ with $x^{n}(\omega)>\omega_{2}>x^{n-1}(\omega)$ and let $h \in A(\Omega)$ such that $h\left(\omega_{1}\right)=\omega$ and $h\left(y\left(\omega_{1}\right)\right)=\omega_{2}$. Thus

$$
x^{n}(\omega)>z(\omega)>x^{n-1}(\omega),
$$

where $z=h y h^{-1}$. It follows that

$$
\left[\left(x^{-(n-1)} z\right) \wedge\left(z^{-1} x^{n}\right)\right]^{+}>1
$$

in $A(\Omega)$. Consider the $l$-homomorphism $f: G \rightarrow A(\Omega)$ extending the inclusion map $f_{1}: G_{1} \rightarrow G(\Omega)$ and the $l$-homomorphism $f_{2}: G_{2} G \rightarrow(\Omega)$ defined by $f_{2}(g)=$ $h g h^{-1}$, for all $g \in G_{2}$. In view of $(*), f\left(s_{n}\right)>1$ in $f(G)$ and hence $s_{n}>1$ in $G$. This completes the proof of the theorem.

The techniques of the present paper are not sufficiently general to settle Problem 2. However, the following partial result can be easily established using Theorem 6 . 
THEOREM 8. If $\mathscr{U}$ is a proper l-group variety and $G_{1}, G_{2}$ are nontriviall-groups in $\mathscr{U}$ with a strong order unit, then $G_{1}^{\mathscr{U}} \cup G_{2}$ has an infinite disjoint set.

PROOF. Every proper variety of $l$-groups is contained in the variety of normal valued $l$-groups (Holland [7]). Hence it is easy to verify that if $G_{1}$ and $G_{2}$ satisfy the conditions stated above, then $G_{1} \neq G_{1}^{\prime}$ and $G_{2} \neq G_{2}^{\prime}$. The conclusion now follows from Theorem 6 .

We finally mention that Theorem 6 implies that for every nontrivial $l$-group variety $\mathscr{U}$, any $\mathscr{U}$-free $l$-group of rank $\geq 2$ contains an infinite disjoint set. This result was first established in [12]. That such an $l$-group satisfies the $\aleph_{1}$-disjointness condition was proved in [2 and 10].

\section{REFERENCES}

1. M. E. Adams and D. Kelly, Disjointness conditions in free products of lattices, Algebra Universalis 7 (1977), 245-258.

2. J. T. Baldwin, J. Berman, A. M. W. Glass and W. Hodges, A combinatorial fact about free algebras, Algebra Universalis 15 (1982), 145-152.

3. A. Bigard, K. Keimel and S. Wolfenstein, Groupes et anneaux réticulés, Springer-Verlag, Berlin, 1977.

4. J. D. Franchello, Sublattices of free products of lattice ordered groups, Algebra Universalis 8 (1978), 101-110.

5. A. M. W. Glass, Ordered permutation groups, London Math. Soc. Lecture Notes Ser., No. 55, Cambridge Univ. Press, London and New York, 1981.

6.

7. W. C. Holland, The lattice ordered group of automorphisms of an ordered set, Michigan Math. J. 10 (1963), 399-408.

8. $\ldots$, The largest proper variety of lattice-ordered groups, Proc. Amer. Math. Soc. 57 (1976), 25-28.

9. R. Botto Mura and A. Rhemtulla, Orderable groups, Marcel Dekker, New York, 1977.

10. W. B. Powell and C. Tsinakis, Free products in the class of abelian l-groups, Pacific J. Math. 104 (1983), 429-442.

11. _ The distributive lattice free product as a sublattice of the abelian l-group free product, J. Austral. Math. Soc. 34 (1983), 92-100.

12. __ Disjointness conditions for free products of lattice ordered groups, Arch. Math. 46 (1986), 491-498.

13. __ Free products of lattice ordered group, Algebra Universalis 18 (1984), 178-198.

14. E. C. Weinberg, Free lattice-ordered abelian groups, Math. Ann. 151 (1963), 187-199.

Department of Mathematics, OKLahoma State University, Stillwater, OKLAHOMA 74078

Department of Mathematics, Vanderbilt University, NashVille, Tennessee 37235 